\title{
Modulation Formats for Beyond-100Gbps Ethernet Optical Links - A Review of Research
}

Jensen, Jesper Bevensee; Iglesias OImedo, Miguel; Tafur Monroy, Idelfonso

Published in:

ACP/IPOC 2013

Publication date:

2013

Document Version

Publisher's PDF, also known as Version of record

Link back to DTU Orbit

Citation (APA):

Jensen, J. B., Iglesias Olmedo, M., \& Tafur Monroy, I. (2013). Modulation Formats for Beyond-100Gbps Ethernet Optical Links - A Review of Research. In ACP/IPOC 2013 [ATh3E.1] Optical Society of America.

\section{General rights}

Copyright and moral rights for the publications made accessible in the public portal are retained by the authors and/or other copyright owners and it is a condition of accessing publications that users recognise and abide by the legal requirements associated with these rights.

- Users may download and print one copy of any publication from the public portal for the purpose of private study or research.

- You may not further distribute the material or use it for any profit-making activity or commercial gain

- You may freely distribute the URL identifying the publication in the public portal

If you believe that this document breaches copyright please contact us providing details, and we will remove access to the work immediately and investigate your claim. 


\title{
Modulation Formats for Beyond-100Gbps Ethernet Optical Links - A Review of Research
}

\author{
Jesper Bevensee Jensen, Miguel Iglesias Olmeido and Idelfonso Tafur Monroy \\ DTU Fotonik, Department of Photonics Engineering, Technical University of Denmark, Ørsteds Pads, Building 343, DK-2800 Kgs. Lyngby, \\ Denmark \\ jebe@fotonik.dtu.dk
}

\begin{abstract}
The current increase in data-centers traffic and cloud-based services presents a formidable challenge for optical interconnects. We examine these challenges, and review recent breakthroughs in advanced modulation formats formats for intensity modulation - direct detection.

OCIS codes: (060.2360) Fiber optics links and subsystems; (060.4250) Networks; (060.4080) Modulation
\end{abstract}

\section{Introduction}

In these years, a chasm is developing between the capacity of core networks and the capacity of optical Ethernet links inside data centers. At the same time, more and more Internet traffic takes place inside data centers due to the increase in use of cloud based services, high definition (HD) TV streaming, on-line gaming and sharing of photos, videos and other files. These two developments combined dictate an increased effort in the development of faster optical Ethernet links. In this paper, we present an overview of recent breakthroughs in advanced modulation of intensity modulated (IM) direct detection (DD) optical links, which potentially can provide the required increase in Ethernet link capacity while maintaining the bandwidth and form factor of existing links.

In the report "IEEE Industry Connections Ethernet Bandwidth Assessment" published by the IEEE 802.3 Ethernet Working Group [1], the Chairman, David Law states that “...the bandwidth requirements for core networking and computing applications were growing at different rates, driving the need to develop the two new wireline Ethernet speeds..." In fact, it is observed that core network bandwidth doubles every 18 months, whereas server and computing application bandwidth doubles every 24 months. This difference in bandwidth growth rates underpins the need for faster Ethernet links. Demand for $100 \mathrm{Gbps}$ Ethernet is foreseen already in 2013 (indeed, the percentage of data center links operating at $100 \mathrm{Gbps}$ has inecreased from below $10 \%$ in 2011 to above $20 \%$ in 2013), and demand for $400 \mathrm{Gbps}$ links is foreseen in 2015 [1]. In addition, there is an emerging need for increasing the reach up to $10 \mathrm{~km}$, forcing future solutions to be robust to dispersion while keeping complexity low.

Current and upcoming standards for $100 \mathrm{Gbps}$, such as 100GBASE-SR10, 100GBASE-SR4, and 100GBASELR4 are based on the use of 10 lanes of $10 \mathrm{Gbps}$ or 4 lanes at $25 \mathrm{Gbps}$ each. Upgrading to $400 \mathrm{Gbps}$ links using the same technology will require an increase in the number of lanes to e.g. 16 lanes operating at $25 \mathrm{Gbps}$ each, thereby making it challenging to meet $400 \mathrm{Gbit} / \mathrm{s}$ form-factor pluggable (e.g. CFP4) requirements on power consumption and footprint [2].

As an alternative, advanced modulation formats capable of higher transmission speeds at reduced bandwidths have been researched in recent years. This paper provides an overview of this research, and presents some of the technological challenges involved with their deployment.

\section{Overview of recent breakthroughs in spectrally efficient IM/DD modulation formats}

Advanced modulation formats capable of transmitting more than one bit per optical symbol have been used and researched for core network applications in several years and today quadrature phase shift keying (QPSK) systems are available commercially. These, however, rely on combined modulation of the optical phase and amplitude, and are thus not suitable for IM/DD links. For data center interconnects, alternative solutions based solely on optical amplitude modulation must be developed.

One of the simplest methods of achieving high spectral efficiency in IM/DD links is to simply increase the number of amplitude levels in the signal. This modulation format is commonly referred to as M-level pulse amplitude modulation (M-PAM), where M refers to the number of levels in the signal. Each optical symbol will carry $\log _{2}(\mathrm{M})$ bits of information. In 2013, 4-PAM modulation of a vertical cavity surface emitting laser (VCSEL) at $50 \mathrm{Gbps}$ was reported [3]. The $50 \mathrm{Gbps}$ signal from the VSCEL is polarization multiplexed to a total capacity of $100 \mathrm{Gbps}$ using a symbol rate of only 25 Gbaud. The signal is transmitted over $100 \mathrm{~m}$ of standard single mode fiber (SSMF). In order to receive the signal error free, custom forward error correction, where the two bit streams in the 
signal are encoded with different FEC codes, is employed. For the least significant bit, 20\% overhead FEC is required to recover the signal. A slightly higher bit rate of $60 \mathrm{Gbps}$ for 4-PAM VCSEL links was reported in 2013 [4], albeit only for $2 \mathrm{~m}$ transmission over multimode fiber. The signal is recovered error free without forward error correction. 4-PAM modulation at 25 Gbaud would enable 400 Gbps Ethernet links employing 8 lanes, and can thus be considered a stepping stone towards 4 lane solutions for $400 \mathrm{Gbps}$ links, which would require 16 amplitude levels for M-PAN signaling; a result which at the moment must be considered very challenging due to the signal to noise ratio (SNR) it would require. On the other hand, M-PAM is comparable simpler to implement that the other modulation formats examined in the remainder of this paper. Additionally, VCSEL drivers for 4-PAM signaling have already been demonstrated [5].

From the M-PAM results described above, it can be concluded that achieving the goal of 4 bits per symbol, or $100 \mathrm{Gbps}$ in less than $25 \mathrm{GHz}$ bandwidth, employing modulation in only one dimension is very challenging. But as Ethernet links relies on IM/DD, we cannot employ the phase of the optical carrier as the second dimension to modulate. This has generated interest in subcarrier modulated quadrature amplitude modulation (QAM), where the laser is driven by a QAM modulated electrical radio frequency (RF) subcarrier. Conventionally, subcarrier QAM modulation requires that the frequency of the subcarrier is equal to or higher than the symbol rate of the data signal. This means that the required bandwidth corresponds to the bandwidth of the double sided signal spectrum so that in order to achieve $100 \mathrm{Gbps}$ in a $25 \mathrm{GHz}$ bandwidth, a $12.5 \mathrm{GHz}$ subcarrier would have to be modulated with a 64 level QAM signal. An interesting alternative consisting of QAM modulation at RF frequencies below the symbol rate has been proposed and demonstrated [6]. The achieved bit rate is 10 Gbps employing 4-QAM modulation of a $5 \mathrm{GHz}$ subcarrier, resulting in a signal bandwidth of $7.5 \mathrm{GHz}$. The sub-cycle QAM signal is generated by delaying and adding the two outputs of an electrical XOR gate whose inputs consists of a non-return-to-zero (NRZ) data signal at the baud rate and an RF carrier at the subcarrier frequency. This method is originally proposed in [7]. The signal is transmitted over $20 \mathrm{~km}$ of SSMF, and display transmission performance and dispersion tolerance performance similar to or better than on-off keying $(\mathrm{OOK})$ at the same bit rate. This result shows the great promise and potential of sub-cycle QAM modulation. In order to achieve $100 \mathrm{Gbps}$, a $12.5 \mathrm{GHz}$ subcarrier could be modulated with a 25 Gbaud 16-QAM signal resulting in a signal bandwidth of approximately $19 \mathrm{GHz}$, i.e. compatible with the requirements for a 4-lane $400 \mathrm{Gbps}$ solution. This, however, has not yet been demonstrated.

Instead of employing a single subcarrier at a very high symbol rate, several subcarriers at a low symbol rate can be combined to form a single signal of high bit rate. Since the bandwidth of each subcarrier is very low, a high spectral efficiency can be maintained without resorting to sub-cycle modulation. Two flavors of this modulation have been demonstrated for IM/DD optical links, namely discrete multitone (DMT) and orthogonal frequency division multiplexing (OFDM). For the latter, it is a requirement that the individual subcarriers are orthogonal to each other. DMT has been demonstrated at $100 \mathrm{Gbps}$ employing wavelength division multiplexing (WDM) of two channels, each operating at $50 \mathrm{Gbps}$ [8]. The signal is generated employing directly modulated lasers with a $3 \mathrm{~dB}$ modulation bandwidth of $16 \mathrm{GHz}$. Transmission over $80 \mathrm{~km}$ of SSMF is achieved in the $1300 \mathrm{~nm}$ wavelength band as well as in the $1550 \mathrm{~nm}$ wavelength band. For the $1550 \mathrm{~nm}$ experiment, several of the subcarriers experience dispersion induced fading, and dispersion compensation is required to recover the signal. The target of $100 \mathrm{Gbps}$ per lane can be realized by doubling the bandwidth of the signal. As the DMT signal is generated in the digital domain employing an arbitrary waveform generator (AWG) with a sampling rate of $34 \mathrm{GSa} / \mathrm{s}$, an AWG or a digital to analog converter (DAC) with a $68 \mathrm{GSa} / \mathrm{s}$ would be required in order to realize $100 \mathrm{Gbps}$ per lane.

DMT is currently the most used modulation format for digital subscriber lines (DSL). Before 1996, however, another modulation format dominated this field, namely carrierless amplitude and phase modulation (CAP). CAP is in many ways similar to QAM described above. It employs two orthogonal dimensions for the modulation of the signal. Contrary to QAM, however, CAP does not use carriers. The orthogonal signals are generated by transversal filters with orthogonal impulse responses. Most commonly, the filters consists of a square-root raised cosine shaping filter multiplied by a sine and a cosine respectively to generate the orthogonal waveforms. Compared to DMT, CAP has the advantage of the possibility of an analog implementation. This is demonstrated at 40 Gbps in [9]. In addition, it is shown that CAP is superior to DMT in terms of SNR requirements and robustness towards multipath interference [10].

A variation of CAP employing several sub bands and dubbed "MultiCAP" is proposed and demonstrated experimentally in [11]. The achieved bit rate is $102 \mathrm{Gbps}$ in a $3 \mathrm{~dB}$ bandwidth of $14 \mathrm{GHz}$. The signal is transmitted over $15 \mathrm{~km}$ of SSMF. The introduction of multiple bands in the CAP signal overcomes the main challenge of CAP signaling: the requirement of a channel with flat frequency response across the signal's frequency spectrum. With MultiCAP, it is straightforward to employ different modulation order in the different sub bands according to the frequency response of the channel. The MultiCAP signal is generated using a DAC operating at a sample rate of 


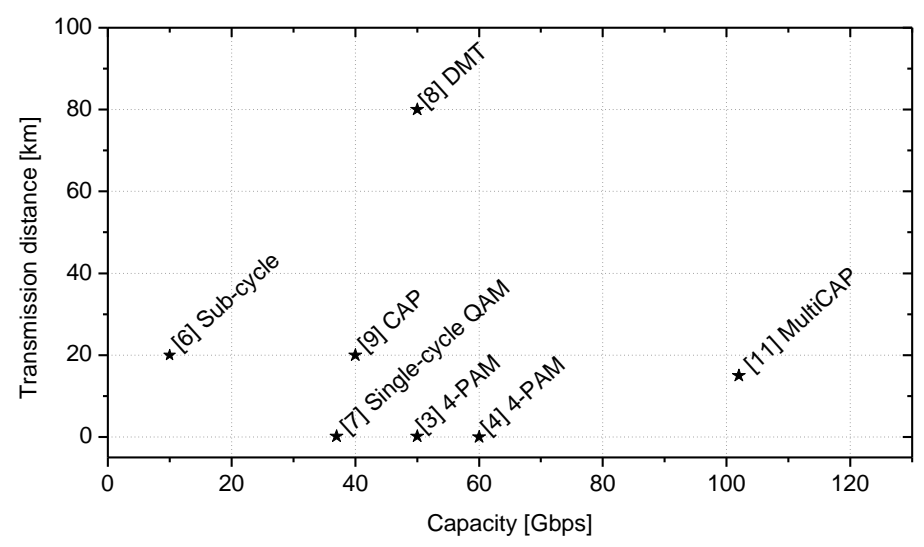

Fig. 1. Capacity and transmission distance achieved in the demonstrations discussed in the paper.

$64 \mathrm{GSa} / \mathrm{s}$, and thus employ a highly demanding digital implementation. Contrary to the DMT case, however, an analog implementation could be realized provided the appropriate transversal filters were designed.

The various examples mentioned above are collected in Fig. 1 in terms of capacity and achieved transmission distance.

\section{Discussions and Conclusions}

400 Gbps optical Ethernet links is expected to be in demand already in 2015. A 4-lane solution, which is compatible with current $100 \mathrm{Gbps}$ 4-lane solutions is very attractive, and will require transmission of 100 Gbps in less than $25 \mathrm{GHz}$ bandwidth. This has been achieved in one of the experimental demonstrations discussed here: The $102 \mathrm{Gbps}$ MultiCAP experiment [11]. The demonstration is realized by employing state-of-the-art digital to analog converters (DACs) and off line digital signal processing. Recent development in the capabilities of DACs and field programmable gate arrays (FPGAs) is indicating that a real time solution could be realistic in just a few years [12]. In order to realize a low cost solution, a number of challenges remain, specifically regarding the development of high speed, cost efficient linear drivers for the laser and linear trans-impedance amplifiers (TIAs) for the photo detector. Additionally, more investigation in power consumption is required.

\section{References}

[1] The IEEE 802.3 Ethernet Working Group, “IEEE Industry Connections Ethernet Bandwidth Assessment," available from http://t.co/vQvUNgtE (2012).

[2] C. Cole, "Beyond 100G client optics", IEEE Communications Magazine, vol. 50, no. 2, pp. s58-s66, Feb. 2012.

[3] R. Rodes, M. Müeller, B. Li, J. Estaran, J. B. Jensen, T. Gruendl, M. Ortsiefer, C. Neumeyr, J. Rosskopf, K. J. Larsen, M.-C. Amann, and I. T. Monroy, "High-Speed $1550 \mathrm{~nm}$ VCSEL Data Transmission Link Employing 25 GBd 4-PAM Modulation and Hard Decision Forward Error Correction," IEEE Journal of Lightwave Technology, vol. 31, no 4, pp. 689-695 (2013)

[4] K. Szczerba, P. Westbergh, M. Karlsson, P.A. Andrekson and A. Larsson, " 60 Gbits error-free 4-PAM operation with 850 nm VCSEL," Electronics Letters, vol. 49, no. 15 (2013)

[5] N. Quadir, P. Ossieur and P. D. Townsend, "A 56Gb/s PAM-4 VCSEL driver circuit", in Proceedings of ISSC 2012 (2012)

[6] T. Pham, R. Rodes, J. B. Jensen, C. J. Chang Hasnain and I. T. Monroy, "Sub-cycle QAM modulation for VCSEL-based optical fiber links", Optics Express, vol. 21, no. 2, pp.1830-1839 (2013).

[7] K. Szczerba, B.-E. Olsson, P. Westbergh, A. Rhodin, J. S. Gustavsson, A. Haglund, M. Karlsson, A. Larsson, and P. A. Andrekson, "37 Gbps transmission over $200 \mathrm{~m}$ of MMF using single cycle subcarrier modulation and a VCSEL with $20 \mathrm{GHz}$ modulation bandwidth," in Proceedings of 36th European Conference on Optical Communication (2010), paper We.7.B.2.

[8] T. Tanaka, M. Nishihara, T. Takahara, L. Li, Z. Tao and J. C. Rasmussen, “Experimental investigation of 100-Gbps transmission over 80-km single mode fiber using discrete multi-tone modulation”, in Proceedings of SPIE 8646N, vol. 8646 (2013).

[9] J. D. Ingham, R. V. Penty, I. H. White and D. G. Cunningham, "40 Gb/s Carrierless Amplitude and Phase Modulation for Low-Cost Optical Datacommunication Links", In Proccedings of 2011 Conference on Optical Fiber Communication - OFC 2011 Collocated National Fiber Optic Engineers Conference OFC/NFOEC 2011, paper OThZ3 (2011).

[10] A. F. Shalash and K. K. Parhi, "Comparison of discrete multitone and carrierless AM/PM techniques for line equalization," in IEEE International Symposium on Circuits and Systems. (ISCAS), 1996, vol. 2, pp. 560-563. (1996)

[11] M. I. Olmedo, T. Zuo, J. B. Jensen, Q. Zhong, X. Xu and I .T. Monroy, “Towards 400GBASE 4-lane Solution Using Direct Detection of MultiCAP Signal in $14 \mathrm{GHz}$ Bandwidth per Lane", In Proceeding of The Optical Fiber Communication Conference and Exposition and the National Fiber Optic Engineers Conference, OFC/NFOEC, paper PDP5C (2012).

[12] M. Gustlin,"Feasibility of a 400GbE PCS” Available from http://www.ieee802.org/3/ad_hoc/hse/public/12_09/gustlin_hse_01_0912.pdf 